\title{
FLUTUAÇÃO POPULACIONAL E SINTOMAS DE DANO POR ÁCAROS (ACARI) EM SERINGUEIRA NO ESTADO DO MATO GROSSO, BRASIL ${ }^{1}$
}

\author{
Noeli Juarez Ferla² e Gilberto José de Moraes ${ }^{3}$
}

\begin{abstract}
RESUMO - Apesar da grande importância da cultura da seringueira (Hevea brasiliensis Muell. Arg.) para o Brasil, pouco se conhece sobre a flutuação populacional dos ácaros nessa cultura. O objetivo deste trabalho foi estudar a flutuação populacional de ácaros na seringueira no Estado do Mato Grosso, bem como observar a evolução dos sintomas de ataque de tais pragas nessa planta. O estudo foi conduzido em campos de seringueira de Itiquira e Pontes e Lacerda, Estado do Mato Grosso, com seis clones: FX 3864, RRIM 600, IAN 873, IAN 713, PB 260 e PR 255, cujas coletas foram realizadas de agosto de 1998 a julho de 2000. Na safra de 1998/ 1999, a amostragem foi de 150 folhas de cada um dos estratos basal, mediano e apical, em 10 plantas ao acaso. A metodologia de coleta na safra de 1999/2000 foi alterada devido aos resultados da parcial anterior de cinco folhas do estrato mediano de cada uma das 15 plantas ao acaso. Calacarus heveae Feres foi a espécie mais frequiente em Itiquira e Phyllocoptruta serigueirae Feres em Pontes e Lacerda. A queda prematura das folhas foi observada apenas em Itiquira.
\end{abstract}

Palavras-chave: Calacarus heveae, Tenuipalpus heveae e Eriophyidae.

\section{POPULATION FLUCTUATION AND MITE (ACARI) DAMAGE SYMPTOMS IN RUBBER TREES IN THE STATE OF MATO GROSSO, BRAZIL}

\begin{abstract}
Despite the importance of rubber trees (Hevea brasiliensis Muell. Arg.) to Brazil, little is known about mite population fluctuation on this crop. The aim of this work was to study the fluctuation of the mite population on rubber trees in the state of Mato Grosso and to observe the evolution of the symptoms caused by their attack. This study was conducted in rubber tree fields at Itiquira and Pontes e Lacerda in the state of Mato Grosso with six clones: FX 3864, RRIM 600, IAN 873, IAN 713, PB 260 and PR 255. Samplings were conducted between August 1998 and July 2000. The 1998/1999 samples consisted of 150 leaves of each of the basal, median and apical strata of 10 plants, randomly taken. The 1999/2000 sampling methodology was changed due the results for the previous partial for five leaves of the median stratum of each of 15 plants randomly taken. Calacarus heveae Feres was the most frequent species in Itiquira and Phyllocoptruta serigueirae Feres in Pontes e Lacerda. Early leaf drop was observed only in Itiquira
\end{abstract}

Keywords: Calacarus heveae, Tenuipalpus heveae and Eriophyidae.

\section{INTRODUÇÃO}

Apesar da grande importância da cultura da seringueira (Hevea brasiliensis Muell. Arg.), pouco se conhece sobre a dinâmica populacional de ácaros considerados pragas nesta cultura, com estudos realizados apenas no Acre, Amazonas e São Paulo (FAZOLIN e PEREIRA, 1989;
FURQUIM, 1994; VIEIRA e GOMES, 1999; FERLA e MORAES, 2002; BELLINI et al., 2005).

Calacarus heveae Feres e Tenuipalpus heveae Baker são consideradas as espécies acarinas mais importantes na cultura da seringueira (FERES, 1992, 2000; FERLA e MORAES, 2002). Calacarus heveae,

\footnotetext{
${ }^{1}$ Recebido em 11.12.2006 e aceito para publicação em 20.02.2008.

${ }^{2}$ Centro Universitário UNIVATES, Lajeado-RS. E-mail: <njferla@ univates.br>.

${ }_{3}^{3}$ Departamento de Entomologia, Fitopatologia e Zoologia Agrícola da Escola Superior de Agricultura Luiz de Queiroz -ESALQ/ USP. E-mail: <gimoraes@esalq.usp.br>.
} 
descrito em seringais do noroeste paulista, prefere a face adaxial dos folíolos (VIEIRA e GOMES, 1999) e causa amarelecimento, bronzeamento, perda do brilho e queda prematura desses folíolos (FERES, 2000). O ataque de $C$. heveae pode provocar níveis de desfolha acima de $75 \%$ no clone RRIM 600 (VIEIRA e GOMES, 1999). Alguns produtores afirmaram que $C$. heveae ocasiona perdas de até $30 \%$ na produção de látex em variedades de $H$. brasiliensis (FERES, 2000). T. heveae predomina na face abaxial dos folíolos, embora, em altas infestações, seja também encontrado na face adaxial (FERES, 2000) e causa queda prematura das folhas.

Os sintomas de ataque e níveis populacionais de Oligonychus gossypii (Zacher) foram relatados nos Estados do Acre e do Amazonas, no período seco do ano, entre outubro e dezembro, caracterizado pelo bronzeamento com tom avermelhado nas duas faces do folíolo (FAZOLIN e PEREIRA, 1989). Folhas de plantas de Eucalyptus sp. atacadas por essa espécie apresentam bronzeamento, encarquilhamento das folhas mais velhas e queda precoce (PEREIRA et al., 2005). Estudos da flutuação de ácaros fitófagos em 10 clones de seringueira no norte do Estado de São Paulo mostraram que $C$. heveae foi mais abundante e com picos populacionais de março a maio (FURQUIM, 1994). Na região noroeste paulista, essa espécie teve pico de abundância no término da estação chuvosa e início da seca, de fevereiro a maio (HERNANDES e FERES, 2006). C. heveae, em Reginópolis, Estado de São Paulo, teve maiores populações no primeiro semestre, coincidindo com o período de maior produção de látex (VIEIRA e GOMES, 1999).

O objetivo deste trabalho foi estudar a flutuação populacional e os sintomas causados por espécies acarinas na cultura de seringueira, no Estado do Mato Grosso.

\section{MATERIAL E MÉTODOS}

O estudo foi conduzido nos seringais das Plantações Edouard Michelin Ltda., no Município de Itiquira (17²13'33.47" S e 54¹0'14.84" O), e do Triângulo Agroindustrial S/A, localizado no Município de Pontes e Lacerda (15'13'08.44' S e 59 20'16.20”' O), ambos no Estado do Mato Grosso.

As coletas foram feitas, mensalmente, em seis clones, sendo escolhidos os mais comuns em cada área. Em Itiquira, foram selecionados os clones PB 260 (4,5 ha e 12 anos) e PR 255 (6,4 ha e 15 anos), enquanto em Pontes e Lacerda os clones IAN 713 (2,6 ha e 15 anos), IAN 873 (17,1 ha e 16 anos), FX 3864 (6,5 ha e 14 anos) e RRIM 600 (3,2 ha e 15 anos). As coletas foram realizadas de agosto de 1998 a julho de 2000, nos clones PB 260 e IAN 873, e nos demais, de agosto de 1999 a julho de 2000.

Os dados da safra 1998/1999 serviram para definir uma metodologia de coleta para acompanhar a dinâmica populacional do ácaro na safra seguinte. Na safra 1998/ 1999, a amostragem consistiu de 15 folhas coletadas de cada uma das 10 plantas escolhidas ao acaso em cada campo, localizadas a pelo menos $20 \mathrm{~m}$ da borda. Foram coletadas três folhas de cada um dos estratos basal, mediano e apical de cada planta, sendo coletada, em cada estrato, uma folha em cada uma das regiões distal, mediana e proximal de cada ramo, totalizando nove folhas. Além disso, foram colhidas três folhas do estrato mediano de cada um dos quadrantes leste e oeste, totalizando seis folhas por planta. Nesses quadrantes, as folhas foram tomadas na região média de ramos da periferia da copa, outra na mesma posição de ramos próximos ao caule e outra na mesma posição em ramos da região intermediária em relação à profundidade da copa de cada uma das plantas amostradas. As parcelas nas quais foram realizadas as amostragens apresentavam aproximadamente 500 plantas com altura aproximada de $20 \mathrm{~m}$. Na segunda safra, entre 1999/2000, cinco folhas de cada uma das 15 plantas escolhidas foram amostradas em ramos da periferia do estrato mediano dos clones IAN 713, IAN 873, FX 3864, PB 260, PR 255 e RRIM 600.

Nenhum tratamento com pesticidas foi realizado durante este trabalho, sendo a última feita cerca de seis meses antes do seu início.

As folhas coletadas foram acondicionadas em sacos de papel e levadas ao laboratório para a contagem das formas móveis sob microscópio estereoscópio, nas duas faces dos folíolos, identificando-os até o nível de família.

As espécies acarinas na safra 1998/1999 foram identificadas de 40 folhas de cada clone, enquanto na safra 1999/2000 foram 20 folhas de cada clone. Até 10 ácaros de cada família foram coletados por folha amostrada. Os eriofídeos foram montados em meio modificado de Berlese para exame ao microscópio (AMRINEe MANSON, 1996) e os demais ácaros, montados em meio de Hoyer (JEPPSON et al., 1975). As lâminas montadas foram mantidas em estufa a $50-60^{\circ} \mathrm{C}$ por cerca de 10 dias, para a fixação, distensão e clarificação dos espécimes e secagem do meio. Posteriormente, foi feita a lutagem das bordas das lamínulas. 
A evolução dos sintomas de ataque dos ácaros à seringueira foi avaliada de acordo com uma escala de notas de 1 a 5 . As folhas sem nenhum dano pertenciam ao nível 1 e aquelas com toda a face superior amarelada, ao nível 5. Os níveis 2, 3 e 4 corresponderam a danos crescentes e intermediários.

\section{RESULTADOS E DISCUSSÃO}

Dinâmica populacional das famílias encontradas. As maiores populações de ácaros, no clone FX 3864, em Pontes e Lacerda, observadas na face abaxial pertenceram à família Eriophyidae (Figura 1). Nesse clone, os eriofídeos apresentaram pico populacional na face adaxial com o aumento da umidade relativa. No mês de março, quando houve maior precipitação, a população de eriofídeos diminuiu nas duas faces dos folíolos. Os ácaros das famílias Cunaxidae e Phytoseiidae foram os predadores mais comumente encontrados.

No clone RRIM 600, em Pontes e Lacerda, os ácaros da família Eriophyidae alcançaram maiores populações com densidades semelhantes nas duas faces dos folíolos. Na face adaxial, o pico populacional desse ácaro ocorreu no mês de março e na face abaxial, em abril (Figura 2). O pico populacional dos eriofídeos da face adaxial foi observado no mês de maior precipitação, quando a umidade relativa também foi alta, enquanto o pico populacional dos eriofídeos da face abaxial aconteceu no mês imediatamente posterior ao de maior precipitação. Os ácaros predadores das famílias Cunaxidae e Phytoseiidae também apresentaram maiores populações em períodos semelhantes aos que ocorreram com o clone FX 3864.

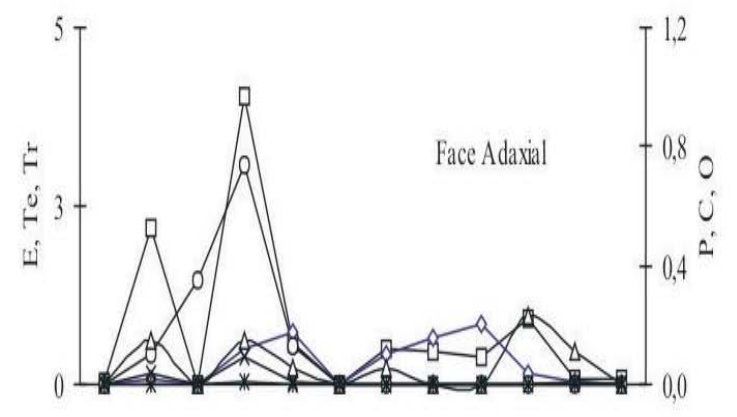

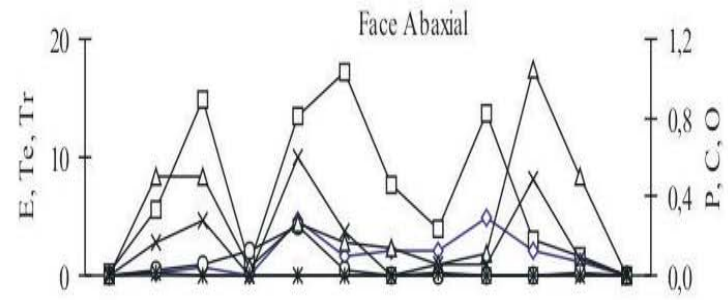

Mês

$$
\rightarrow-E \rightarrow-T e-O-T r-\neg-P * C-0
$$

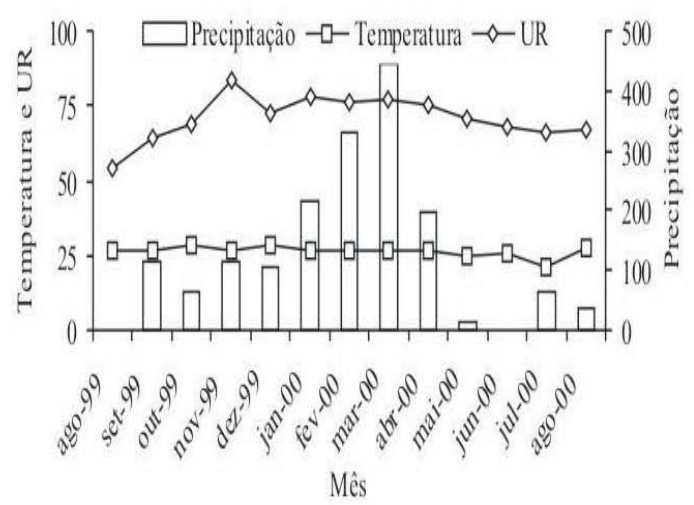

Figura 1 - Flutuação populacional (ácaros/folha) de ácaros no clone FX 3864 de seringueira em Pontes e Lacerda, Mato Grosso ( $\mathrm{E}=$ Eriophyidae; $\mathrm{Te}=$ Tenuipalpidae; $\mathrm{Tr}=$ Tetranychidae; $\mathrm{P}=$ Phytoseiidae; $\mathrm{C}=\mathrm{Cunaxidae} ; \mathrm{e} \mathrm{O}=\mathrm{Acaridae}$, Cheyletidae, Tarsonemidae, Tydeidae, Stigmaeidae e Wintershmidtiidae).

Figure 1-Mite population fluctuation (mites/leaf) on the rubber tree clone FX 3864 in Pontes e Lacerda, Mato Grosso. 

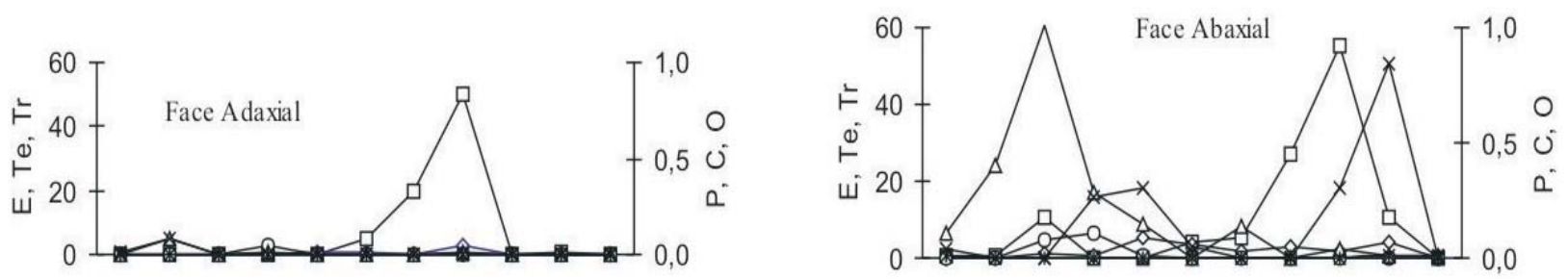

$$
\rightarrow-E \multimap-T e \rightarrow T r \rightarrow P \rightarrow C \rightarrow O
$$

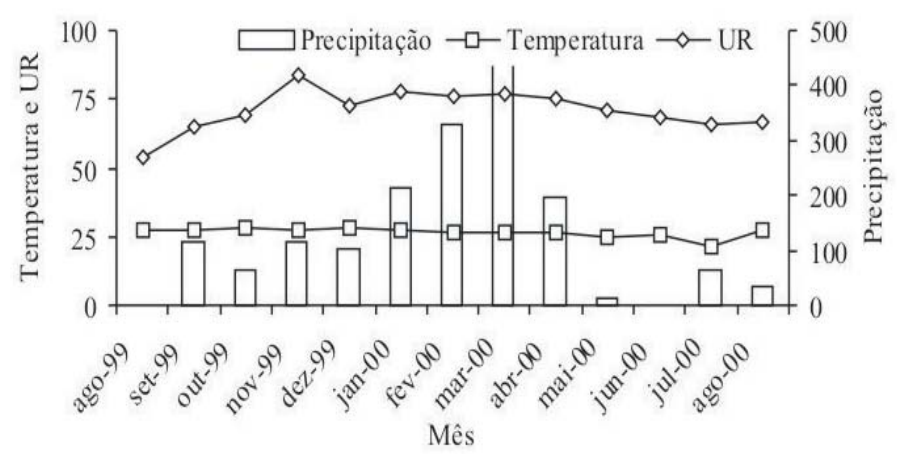

Figura 2 - Flutuação populacional (ácaros/folha) de ácaros no clone RRIM 600 de seringueira em Pontes e Lacerda, Mato Grosso $(\mathrm{E}=$ Eriophyidae; $\mathrm{Te}=$ Tenuipalpidae; $\mathrm{Tr}=$ Tetranychidae; $\mathrm{P}=$ Phytoseiidae; $\mathrm{C}=$ Cunaxidae; $\mathrm{e} \mathrm{O}=\mathrm{Acaridae}$, Cheyletidae, Tarsonemidae, Tydeidae, Stigmaeidae e Wintershmidtiidae).

Figure 2 - Mite population fluctuation (mites/leaf) on the rubber tree clone RRIM 600 in Pontes e Lacerda, Mato Grosso.

No clone IAN 873, em Pontes e Lacerda, os eriofídeos apresentaram maiores populações na face abaxial, a partir de setembro de 1999 (Figura 3). As populações de eriofídeos na face abaxial diminuíram em março e abril de 2000, quando a precipitação foi maior. O aumento da população de $T$. heveae nesse período provavelmente afetou o crescimento das populações de eriofídeos. Os fitoseídeos foram os ácaros predadores mais comuns, mas em outubro de 1998 os tideídeos dos gêneros Pronematus e Parapronematus foram os ácaros predadores mais comuns, enquanto Pseudobonzia sp. e Agistemus floridanus Gonzalez apresentaram altas populações em maio de 1999.

A população de eriofídeos do clone IAN 713, em Pontes e Lacerda, foram maiores na face abaxial, enquanto os tetraniquídeos apresentaram populações semelhantes nas duas faces da folha (Figura 4). As populações de eriofídeos foram maiores na face abaxial em março, quando se observou maior precipitação. O pico populacional dos tetraniquídeos aconteceu no mês de novembro, quando foi observada maior umidade relativa. Os fitoseídeos e os cunaxídeos foram os ácaros predadores mais comuns, com níveis mais elevados de outubro a dezembro de 1999, quando os tetraniquídeos também foram encontrados em níveis mais elevados.

As populações de eriofídeos foram maiores na face adaxial de folhas do clone PB 260, em Itiquira. Os tenuipalpídeos ocorreram em maiores populações na face abaxial (Figura 5). Nos dois anos, as maiores populações de eriofídeos e tenuipalpídeos foram associadas à alta umidade relativa e alta precipitação. Nesse clone, os estigmeídeos e os fitoseídeos foram os ácaros predadores mais comuns. Os Stigmaeidae tiveram maiores populações em novembro de 1998 , janeiro de 1999, novembro de 1999 e janeiro de 2000, quando as populações de $C$. heveae foram mais altas na face adaxial e as de $T$. heveae, na face abaxial.

De forma semelhante ao registrado no clone PB 260, os eriofídeos alcançaram maiores populações no clone PR 255 na face adaxial em Itiquira (Figura 6). As maiores populações de eriofídeos foram verificadas no mês de maior precipitação e alta umidade relativa. Nesse clone, os estigmeídeos foram os ácaros 
predadores mais comuns. A. floridanus apresenta oviposição relativamente alta em comparação com $C$. heveae e T. heveae (FERLA e MORAES, 2003). A introdução inoculativa ou a utilização de práticas que preservem essa espécie no campo seriam estratégias recomendáveis para manter esse predador no campo.

As possíveis razões para a abundância de eriofídeos na face adaxial das folhas em Itiquira e na face abaxial em Pontes e Lacerda foram as condições ambientais diferentes entre os locais amostrados. A disponibilidade de água e as variações de temperatura entre as estações influenciam a disponibilidade de carboidratos na planta (LIMA et al., 2002), o que pode afetar as populações de ácaros fitófagos e predadores. A baixa variação na temperatura impossibilitou a observação desse fator sobre as populações acarinas nos clones.

Populações de ácaros predadores das famílias Cunaxidae, Phytoseiidae e Stigmaeidae estiveram presentes quando da diminuição nos níveis populacionais de $C$. heveae em alguns clones observados, mas, como não foram verificados em todos os clones, não foi possível a formulação de hipótese consistente para explicar essa diminuição populacional de $C$. heveae.

Itiquira, com $C$. heveae sendo a espécie mais abundante, teve a umidade relativa e a precipitação pouco maiores que as do Município de Pontes e Lacerda, onde $P$. seringueirae foi mais abundante. Entretanto, os picos populacionais dessas espécies não podem ser explicados, apenas, pela umidade relativa e precipitação, pois os clones tiveram os picos populacionais em momentos distintos, principalmente com relação a $P$. seringueirae em Pontes e Lacerda. Os picos populacionais de $C$. heveae não tiveram correlação com as condições ambientais, pois foram observados dois momentos de alta precipitação em Itiquira, e somente num dos picos de precipitação foram vistas altas populações dessa espécie. Além disso, a resistência diferenciada por cada clone pode ter influenciado os picos populacionais dessas espécies.
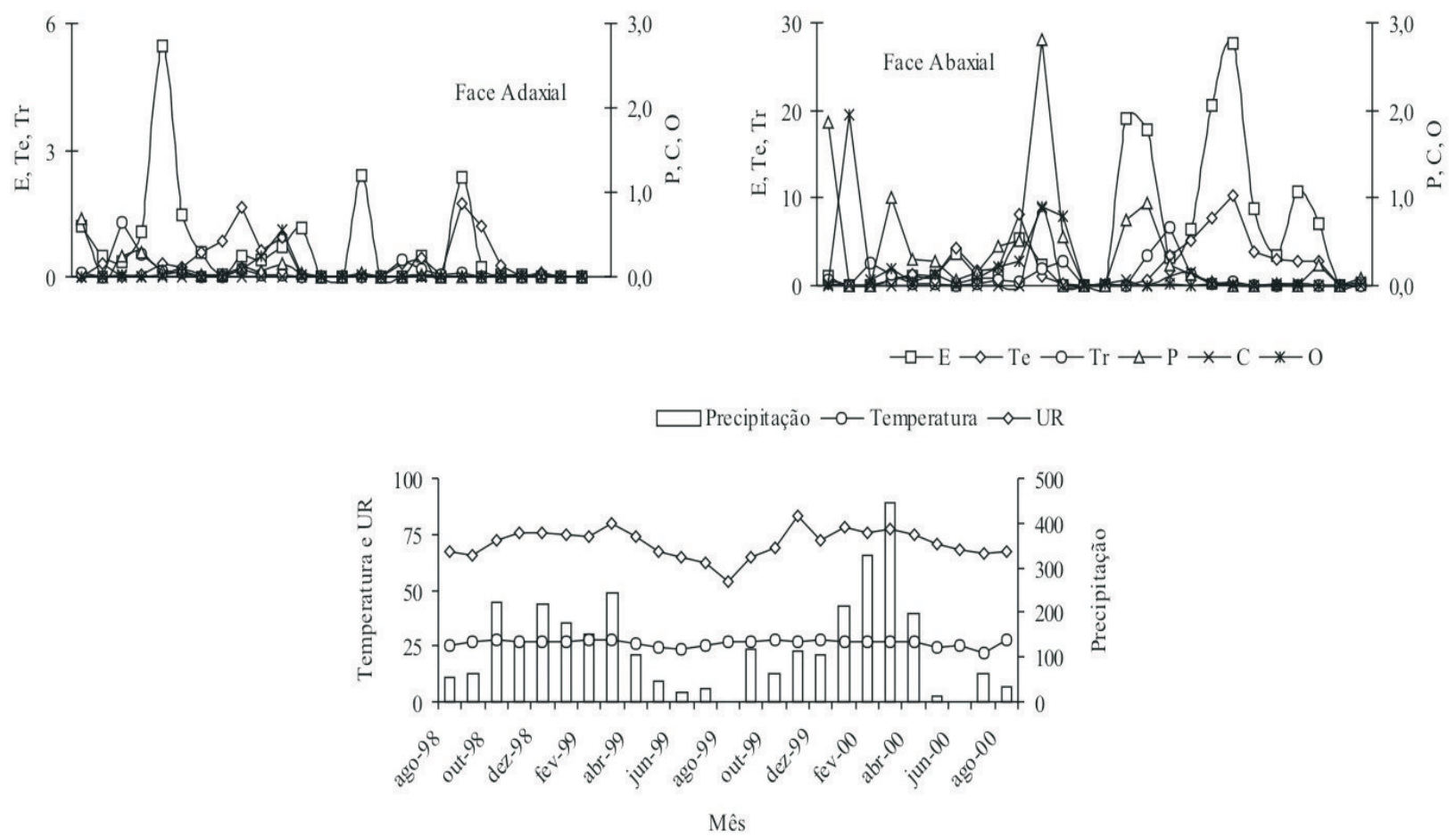

Figura 3 - Flutuação populacional (ácaros/folha) de ácaros no clone IAN 873 de seringueira em Pontes e Lacerda, Mato Grosso $(\mathrm{E}=$ Eriophyidae; $\mathrm{Te}=$ Tenuipalpidae; $\mathrm{Tr}=$ Tetranychidae; $\mathrm{P}=$ Phytoseiidae; $\mathrm{C}=$ Cunaxidae; $\mathrm{O}=\mathrm{O}$ Acaridae, Cheyletidae, Tarsonemidae, Tydeidae, Stigmaeidae e Wintershmidtiidae).

Figure 3 - Mite population fluctuation (mites/leaf) on the rubber tree clone IAN 873 in Pontes e Lacerda, Mato Grosso. 

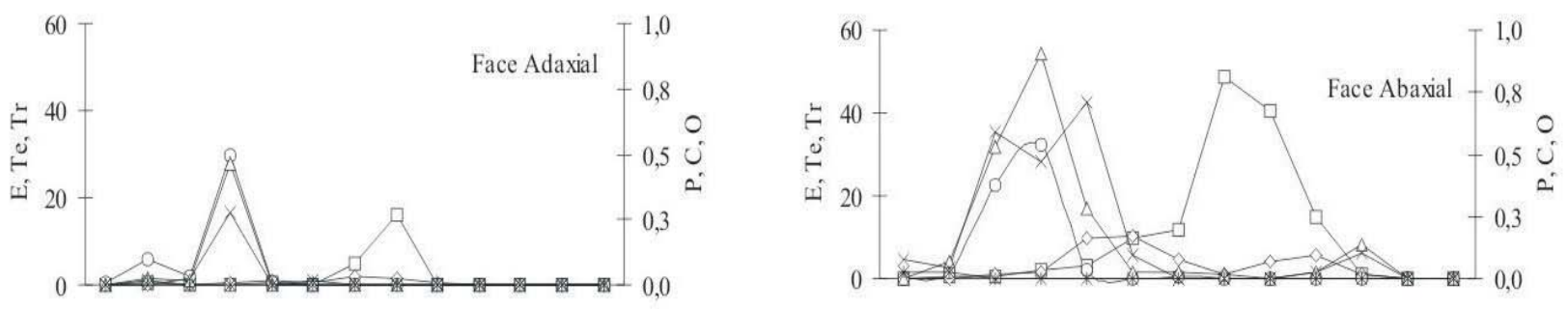

$\rightarrow \square-\mathrm{E} \rightarrow-\mathrm{Te} \rightarrow \mathrm{O}-\mathrm{Tr} \rightarrow-\mathrm{P} \rightarrow \mathrm{C} \rightarrow \mathrm{O}$

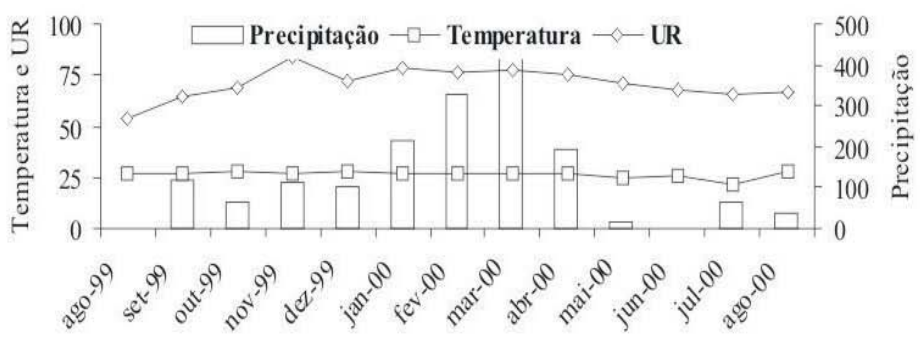

Mês

Figura 4 - Flutuação populacional (ácaros/folha) de ácaros no clone IAN 713 de seringueira em Pontes e Lacerda, Mato Grosso $(\mathrm{E}=$ Eriophyidae; $\mathrm{Te}=$ Tenuipalpidae; $\mathrm{Tr}=$ Tetranychidae $\mathrm{P}=$ Phytoseiidae $; \mathrm{C}=$ Cunaxidae $; \mathrm{e} \mathrm{O}=$ Acaridae, Cheyletidae, Tarsonemidae, Tydeidae, Stigmaeidae e Wintershmidtiidae).

Figure 4-Mite population fluctuation (mites/leaf) on the rubber tree clone IAN 713 in Pontes e Lacerda, Mato Grosso.
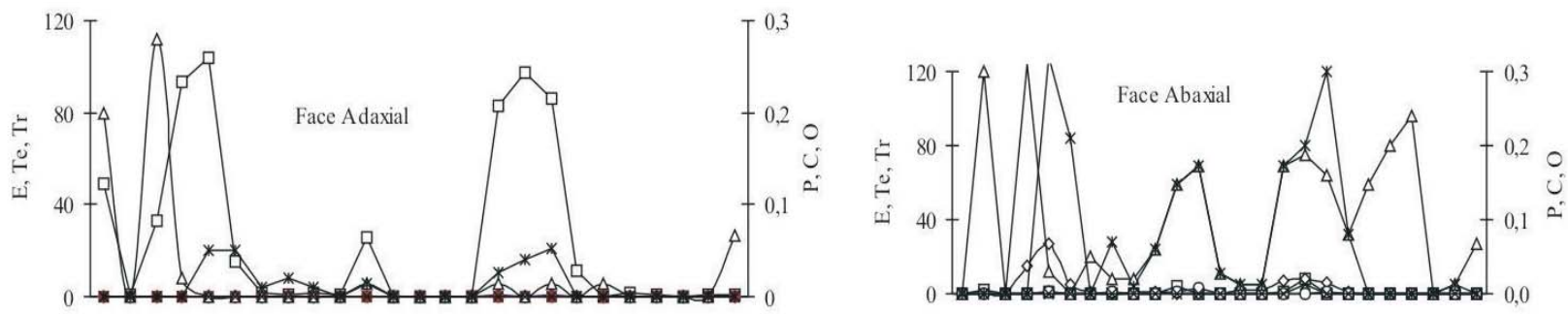

$\rightarrow-\mathrm{E} \rightarrow \mathrm{Te} \rightarrow \mathrm{O}-\mathrm{Tr} \rightarrow \mathrm{P} \rightarrow$ * $\mathrm{C} \rightarrow \mathrm{O}$

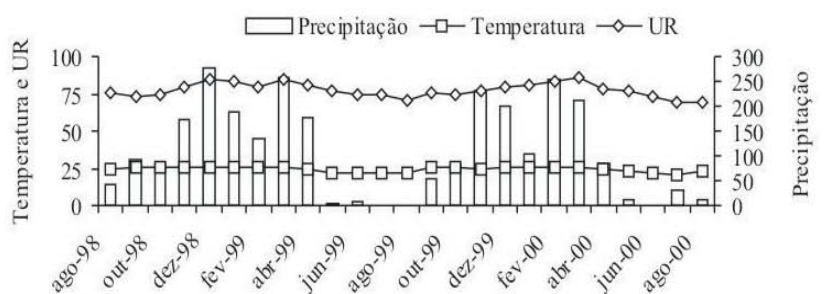

Mês

Figura 5 - Flutuação populacional (ácaros/folha) de ácaros no clone PB 260 de seringueira em Itiquira, Mato Grosso (E $=$ Eriophyidae $; \mathrm{Te}=$ Tenuipalpidae $; \mathrm{Tr}=$ Tetranychidae $\mathrm{P}=$ Phytoseiidae $; \mathrm{C}=$ Cunaxidae; $\mathrm{e} \mathrm{O}=$ Acaridae, Cheyletidae, Tarsonemidae, Tydeidae, Stigmaeidae e Wintershmidtiidae).

Figure 5 - Mite population fluctuation (mites/leaf) on the rubber tree clone PB 260 in Pontes e Lacerda, Mato Grosso.

R. Árvore, Viçosa-MG, v.32, n.2, p.365-376, 2008 

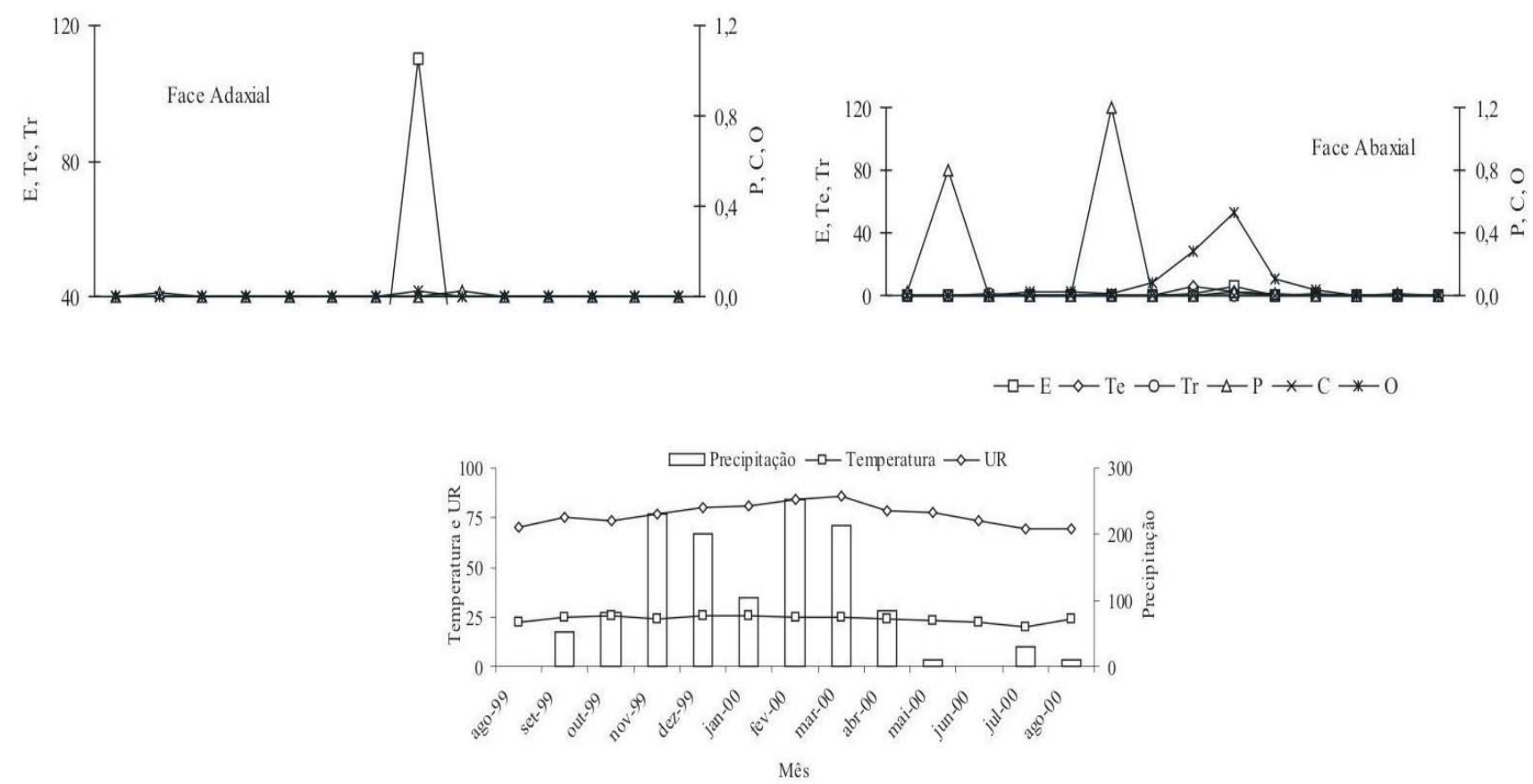

Figura 6 - Flutuação populacional (ácaros/folha) de ácaros no clone PR 255 de seringueira em Itiquira, Mato Grosso (E $=$ Eriophyidae $; \mathrm{Te}=$ Tenuipalpidae $; \mathrm{Tr}=$ Tetranychidae $\mathrm{P}=$ Phytoseiidae $; \mathrm{C}=$ Cunaxidae $; \mathrm{e} \mathrm{O}=$ Acaridae, Cheyletidae, Tarsonemidae, Tydeidae, Stigmaeidae e Wintershmidtiidae).

Figure 6 - Mite population fluctuation (mites/leaf) on the rubber tree clonePR 255 in Pontes e Lacerda, Mato Grosso.

O fungo patogênico Hirsutella thompsonii Fisher foi encontrado geralmente infectando C. heveae, quando este ácaro alcançou altos níveis populacionais no campo. Grande número de ninfas e adultos de $C$. heveae foram mortos por esse fungo em plantações de seringueira no Estado do Mato Grosso (TANZINI et al., 2000). É possível que esse patógeno tenha sido pelo menos um dos responsáveis pelas quedas abruptas das populações de $C$. heveae em Pontes e Lacerda. Entretanto, o efeito sobre $C$. heveae não foi significativo em Itiquira em 2000, pois a redução populacional de $C$. heveae nos dois clones avaliados se deveu, aparentemente, às condições inadequadas das folhas para maior aumento da população do ácaro.

Espécies acarinas mais comuns. Phyllocoptruta seringueirae Feres é o eriofídeo coletado em maior frequiência em Pontes e Lacerda (33\%), enquanto C. heveae foi encontrado em maior frequiência em Itiquira (25,7\%) (Tabela 1). T. heveae apresentou maior número de indivíduos em Pontes e Lacerda $(35,1 \%)$ que em Itiquira (24,8\%). O. gossypii e Teytranychus mexicanus (McGregor) foram os tetraniquídeos com maior número de indivíduos. O. gossypii foi mais freqüente em Pontes e Lacerda $(6,2 \%)$, e T. mexicanus teve freqüência maior em Itiquira $(5,1 \%)$.

Euseius concordis (Chant) e Neoseiulus anonymus (Chant \& Baker) foram as espécies mais abundantes de Phytoseiidae. E. concordis foi mais abundante em Pontes e Lacerda (8,0\%) que em Itiquira (4,6\%), e $N$. anonymus o foi mais em Itiquira $(4,5 \%)$ que naquele município (1,0\%). Já Pseudobonzia sp. foi mais abundante em Pontes e Lacerda (4,7\%). Não foi possível chegarse à conclusão quanto ao tipo de presa preferida por Pseudobonzia sp. Entretanto, nas observações preliminares de laboratório percebeu-se que essa espécie se alimenta de $T$. heveae, e a frequiência de A. floridanus foi maior em Itiquira $(5,4 \%)$ que em Pontes e Lacerda $(0,5 \%)$.

Prevalências de espécies de Eriophyidae e Phytoseiidae. $P$. seringueirae foi a espécie de eriofídeo dominante em Pontes e Lacerda durante todo o estudo, sendo essa dominância nos clones FX 3864 e IAN 713 (Figura 7). C. heveae foi a segunda espécie mais numerosa, especialmente no clone IAN 873, de novembro de 1998 a março de 1999; e RRIM 600, de dezembro de 1999 e março de 2000. S. petiolula teve 
menor freqüência. Em Itiquira, foi dominante $C$. heveae, enquanto $P$. seringueirae e $S$. petiolula foram menos frequientes, exceto em algumas coletas.

E. concordis foi a espécie dominante nos clones FX 3864, RRIM 600 e IAN 713 em Pontes e Lacerda, com maior frequiência de agosto a dezembro (Figura 8). N. anonymus foi mais freqüente nos clones RRIM $600 \mathrm{e}$ IAN 873, de setembro a fevereiro. O total de indivíduos dos outros fitoseídeos foi maior que os de E. concordis e $N$. anonymus no clone FX 3864, de setembro a fevereiro. Em Itiquira, a freqüência de $N$. anonymus foi maior no clone PR 255, entre outubro e fevereiro, enquanto as freqüências de E. concordis e $N$. anonymus foram semelhantes no clone PB 260. Neste, N. anonymus foi mais freqüente de novembro a janeiro, enquanto $E$. concordis o foi nos demais meses.

Tabela 1 - Proporção (\%) das espécies acarinas encontradas em plantações de seringueira nos Municípios de Itiquira e Pontes e Lacerda, Mato Grosso, entre setembro de 1998 e julho de 2000

Table 1 - Proportion (\%) of mite species on rubber trees in Itiquira and Pontes e Lacerda, Mato Grosso, between September 1998 and July 2000

\begin{tabular}{|c|c|c|c|}
\hline Família & Espécie & Itiquira & Pontes e Lacerda \\
\hline & ORDEM MESOSTIGMATA & & \\
\hline \multirow[t]{16}{*}{ Phytoseiidae } & Amblyseius acalyphus Denmark \& Muma & 2,2 & 0,2 \\
\hline & Amblyseius neochiapensis Lofego, Moraes \& McMurtry & 0,08 & 1,3 \\
\hline & Euseius alatus DeLeon & 0,08 & 1,9 \\
\hline & Euseius citrifolius Denmark \& Muma & 2,1 & 0,0 \\
\hline & Euseius concordis (Chant) & 4,6 & 8,0 \\
\hline & Galendromimus alveolaris (DeLeon) & 0,0 & 0,06 \\
\hline & Galendromus annectens DeLeon & 0,1 & 0,2 \\
\hline & Metaseiulus ferlai Moraes. McMurtry \& Lopes & 0,0 & 0,3 \\
\hline & Neoseiulus anonymus (Chant \& Baker) & 1,0 & 4,5 \\
\hline & Neoseiulus tunus (DeLeon) & 0,0 & 0,03 \\
\hline & Typhlodromalus aff. horatii & 0,0 & 0,03 \\
\hline & Typhlodromalus feresi Lofego, Moraes \& McMurtry & 0,0 & 0,03 \\
\hline & Typhlodromips amilus DeLeon & 0,0 & 0,8 \\
\hline & Typhlodromips aff. sinensis & 0,0 & 0,2 \\
\hline & Typhlodromus transvaalensis (Nesbitt) & 0,0 & 0,03 \\
\hline & ORDEM PROSTIGMATA & & \\
\hline Cheyletidae & Hemicheyletia wellsi (Baker) & 0,08 & 0,03 \\
\hline Cunaxidae & Pseudobonzia sp. & 0,1 & 4,7 \\
\hline \multirow[t]{5}{*}{ Eriophyidae } & Aff. Acaphyllisa sp. & 0,08 & 0,0 \\
\hline & Aff. Chakrabartiella sp. & 0,0 & 0,1 \\
\hline & Calacarus heveae Feres & 25,1 & 7,7 \\
\hline & Phyllocoptruta seringueirae Feres & 1,8 & 33,0 \\
\hline & Shevtchenkella petiolula Feres & 0,4 & 1,9 \\
\hline \multirow[t]{2}{*}{ Stigmaeidae } & Agistemus floridanus Gonzalez & 5,4 & 0,5 \\
\hline & Zetzellia quasagistemas Hernandes \& Feres & 0,08 & 0,09 \\
\hline \multirow[t]{2}{*}{ Tarsonemidae } & Polyphagotarsonemus latus (Banks) & 0,08 & 0,1 \\
\hline & Tarsonemus sp. & 2,4 & 0,06 \\
\hline \multirow[t]{2}{*}{ Tenuipalpidae } & Brevipalpus phoenicis (Geijskes) & 0,1 & 0,06 \\
\hline & Tenuipalpus heveae Baker & 35,1 & 24,8 \\
\hline \multirow[t]{5}{*}{ Tetranychidae } & Eutetranychus banksi (McGregor) & 0,1 & 0,0 \\
\hline & Mononychellus sp. & 0,5 & 0,7 \\
\hline & Oligonychus coffeae (Nietner) & 0,4 & 0,7 \\
\hline & Oligonychus gossypii (Zacher) & 3,9 & 6,2 \\
\hline & Tetranychus mexicanus (McGregor) & 5,1 & 2,5 \\
\hline \multirow[t]{5}{*}{ Tydeidae } & Lorryia formosa Cooreman & 1,5 & 0,03 \\
\hline & Lorryia sp. & 1,2 & 0,7 \\
\hline & Parapronematus sp. & 0,3 & 1,3 \\
\hline & Pronematus sp. & 0,08 & 0,5 \\
\hline & ORDEM ASTIGMATA & & \\
\hline \multirow{2}{*}{ Acaridae } & Tyrophagus sp. & 0,3 & 0,0 \\
\hline & Neotropacarus sp. & 0,8 & 0,0 \\
\hline Winterschmidtiidae & Oulenzia sp. & 0,3 & 0,09 \\
\hline
\end{tabular}

R. Árvore, Viçosa-MG, v.32, n.2, p.365-376, 2008 

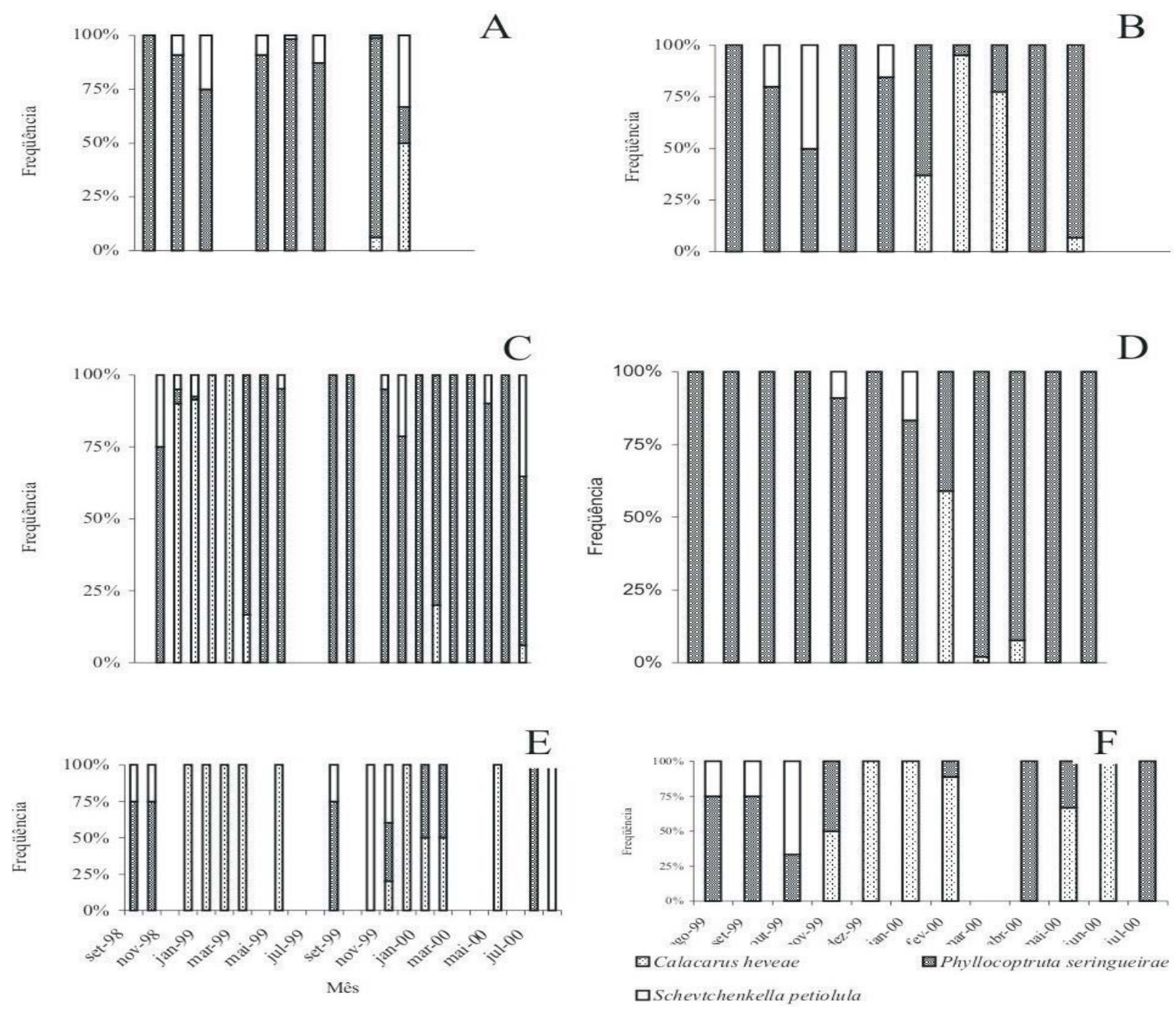

Figura 7 - Freqüência da ocorrência das principais espécies de Eriophyidae nos clones de seringueira avaliados nos Municípios de Pontes e Lacerda (A = clone FX 3864; B = clone RRIM 600; C = clone IAN 873; e D = clone IAN 713) and Itiquira $(\mathrm{E}=$ clone PB 260; e F = clone PR 255).

Figure 7 - Frequency of occurrence of the main species of Eriophyidae in rubber tree clones in Pontes e Lacerda and Itiquira, Mato Grosso.

E. concordis esteve presente durante todo o ano, mas não teve correlação com nenhuma presa específica. Entretanto, essa espécie apresentou maior oviposição quando alimentada com pólen de taboa (Typha angustifolia L.) que com diferentes espécies de ácaros que ocorrem na seringueira (FERLA e MORAES, 2003). Os fitoseídeos do gênero Euseius pertencem a um grupo que, de maneira geral, preferem diferentes tipos de pólen como alimento (McMURTRY e CROFT, 1997). Por isso, os fitoseídeos são responsáveis pela manutenção de populações de tetraniquídeos em baixos níveis, principalmente em plantas perenes, sendo importantes em estratégias de conservação.
Sua ação se dá, usualmente, evitando o aumento de uma população de ácaros fitófagos emestágio inicial de crescimento.

N. anonymus possivelmente tenha suas populações relacionadas com as populações de tetraniquídeos por ter apresentado maior frequiência quando esses ácaros estiveram presentes. Essa hipótese é suportada por Ferla e Moraes (2003), com maior oviposição de N. anonymus quando alimentado com tetraniquídeos do que com outros grupos de ácaros. Essa espécie pertence ao grupo de predadores seletivos, por se alimentarem, preponderantemente, de ácaros da família Tetranychidae (McMURTRY e CROFT, 1997).

R. Árvore, Viçosa-MG, v.32, n.2, p.365-376, 2008 
A

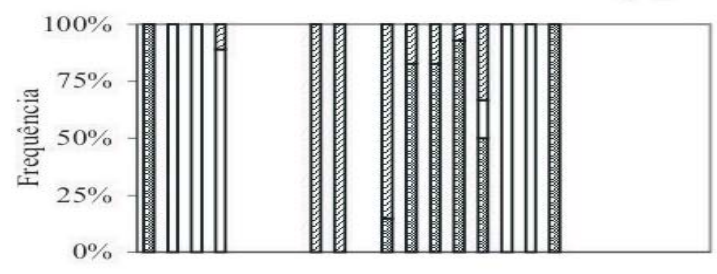

C

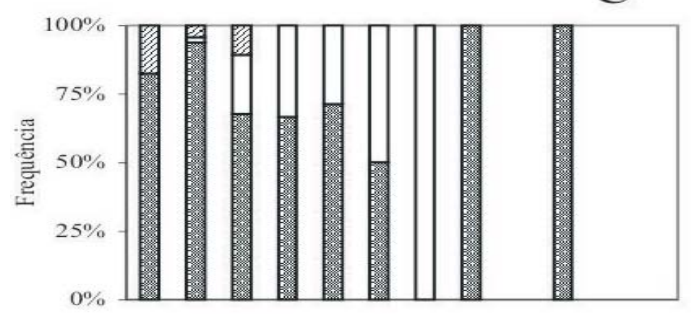

$\mathrm{E}$

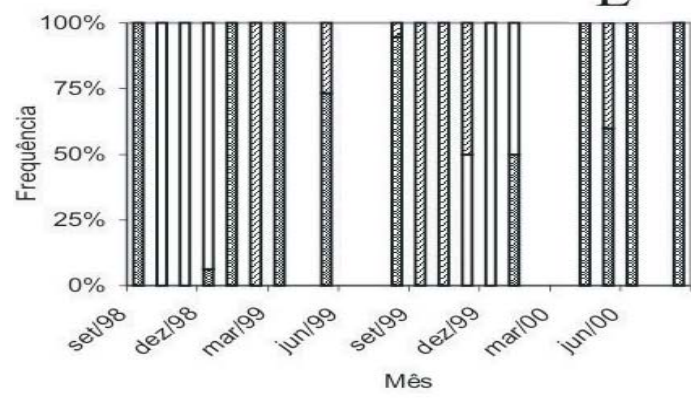

Euseius concordis

$\square$ Neoseiulus anonymus

曰

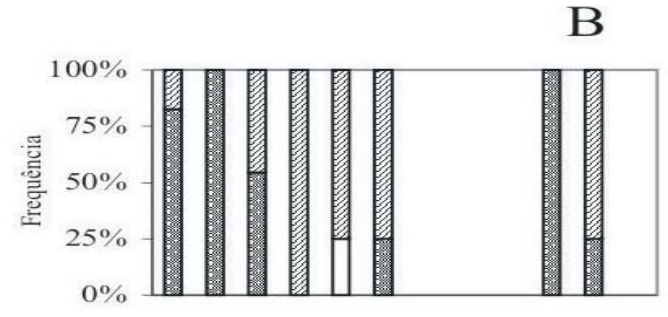

$\mathrm{D}$

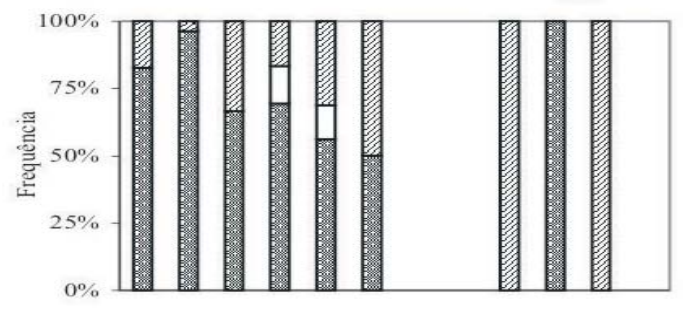

$\mathrm{F}$

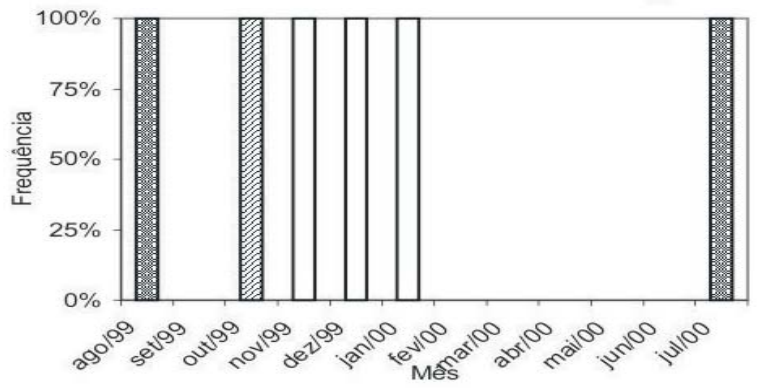

Figura 8 - Freqüência da ocorrência das principais espécies de Phytoseiidae nos clones de seringueira avaliados nos Municípios de Pontes e Lacerda (A = clone FX 3864; B = clone RRIM 600; C = IAN 873; e D = clone IAN 713) e Itiquira $(\mathrm{E}=$ clone $\mathrm{PB} 260 ;$ e $\mathrm{F}=$ clone $\mathrm{PR} 255)$.

Figure 8 -Frequency of occurrence of the main Phytoseiidae species on rubber tree clones in Pontes e Lacerda and Itiquira.

Dano causado e sintomas de ataque. Em Itiquira, o nível máximo de dano foi de 4,5 entre maio e junho no clone PB 260 e quatro no clone PR 255, com queda prematura de folhas a partir de abril (Figura 9). Em Pontes e Lacerda, o nível máximo observado foi 2, nos meses de maio e junho, sem queda prematura de folhas nos clones avaliados.

A queda das folhas das plantas de seringueira acontece naturalmente no início do inverno nos clones observados, no Estado do Mato Grosso. A ausência de um clonecontrole para comparar com os demais clones estudados dificultou a diferenciação do dano observado em Itiquira pela queda natural das folhas. De qualquer forma, independentemente da queda prematura, os danos nos clones PB 260 e PR 255 são bastante significativos, o que indica a importância de $C$. heveae e $T$. heveae. Entretanto, como em Pontes e Lacerda foram observadas apenas altas populações de $P$. seringueirae e como os danos às folhas foram relativamente nessa região, é possível que essa espécie não cause maiores danos aos clones estudados. As populações de eriofídeos foram menores que aqueles danos por $C$. heveae nesse clone no Município de Reginópolis, Estado de São Paulo (VIEIRA e GOMES, 1999). A desfolha provocada por C. heveae no Estado de São Paulo, no clone RRIM600, foi acima de 75\%, enquanto em Pontes e Lacerda a queda não foi significativa. 


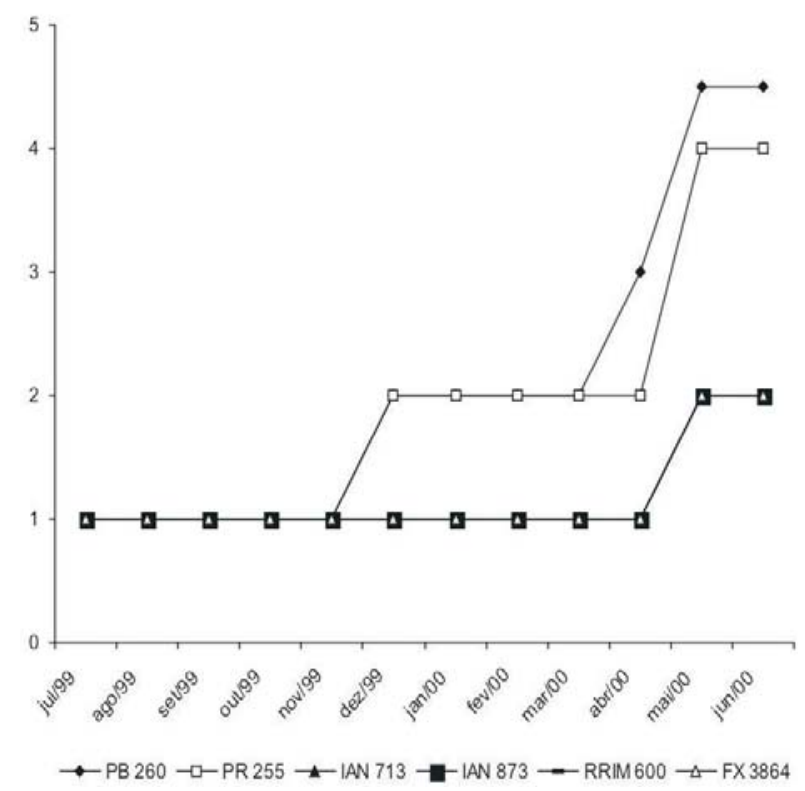

Figura 9-Danos provocados por ácaros em folhas de seringueira entre agosto de 1999 e julho de 2000 , nos clones PB 260 e PR 255 em Itiquira e nos clones IAN 713, IAN 873, RRIM 600 e FX 3864 em Pontes e Lacerda, Estado do Mato Grosso.

Figure 9 -Damage caused by mites to rubber tree leaves between August 1999 and July 2000 on the clones $P B 260$ and PR 255 in Itiquira, and IAN 713, IAN 873, RRIM 600 and FX 3864 in Pontes e Lacerda, Mato Grosso.

\section{CONLUSÕES}

$\mathrm{Na}$ cultura da seringueira no Estado do Mato Grosso, C. heveae, $P$. seringueirae e $T$. heveae são os ácaros fitófagos mais importantes, enquanto no grupo dos predadores se destacam A. floridanus, $E$. concordis e N. anonymus.

Os clones PB 260 e PR 255 apresentaram maiores populações de $C$. heveae e danos mais significativos nas folhas que os demais clones avaliados, enquanto o clone PB 260 exibiu queda prematura das folhas.

\section{AGRADECIMENTOS}

Às empresas Plantações Edouard Michellin Ltda. e Triângulo Agro-Industrial Ltda., pelo financiamento do projeto; aos Engenheiros-Agrônomos Caio Franchechi, Cássio Scomparin e Etiéne Grallien; ao Técnico Agrícola Nilson de Souza, pelo auxílio na realização destes estudos; e ao CNPq, pela concessão de bolsa ao primeiro autor para a realização do doutorado.

\section{REFERÊNCIAS}

AMRINE JR., J. W.; MANSON, D. C. M.

Preparation, mounting and descriptive study of eriophyoid mites. In: LINDQUIST, E. E.; SABELIS, M.W.; BRUIN, J. (Eds.), Eriophyoid mites. Their Biology, Natural Enemies and Control. Amsterdan: Elsevier, 1996. p.383-396.

BELLINI, M. R.; MORAES, G. J.; FERES, R. J. F. Ácaros (Acari) de dois sistemas de cultivo da seringueira no noroeste do Estado de São Paulo. Neotropical Entomology, v.34, n.3, p.475-484, 2005.

FAZOLIN, M.; PEREIRA. L. V. Ocorrência de Oligonychus gossypii (Zacher, 1920) (Acari: Tetranychidae) em seringueiras cultivadas. Anais da Sociedade Entomológica do Brasil, v.18, n.1, p.199-202, 1989.

FERES, R. J. F. A new species of Calacarus Keifer (Acari, Eriophyidae, Phyllocoptinae) from Hevea brasiliensis Muell. Arg. (Euphorbiaceae) from Brazil. International Journal of Acarology, v.18, n.1, p.61-65, 1992.

FERES, R. J. F. Levantamento e observações naturalísticas da acarofauna (Acari, Arachnida) de seringueiras cultivadas (Hevea spp.,

Euphorbiaceae) no Brasil. Revista Brasileira de Zoologia, v. 17, n.1, p.157-173, 2000.

FERLA, N. J.; MORAES. G. J. Ácaros (Arachnida, Acari) da seringueira (Hevea brasiliensis Muell. Arg.) no Estado do Mato Grosso, Brasil. Revista Brasileira de Zoologia, v.19, n.3, p.867-888, 2002.

FERLA, N. J.; MORAES. G. J. Oviposição dos ácaros predadores Agistemus floridanus Gonzalez, Eusieus concordis (Chant) e Neoseiulus anonymus (Chant \& Baker) (Acari) em resposta a diferentes tipos de alimento. Revista Brasileira de Zoologia, v.20, n.1, p.153-155, 2003.

FURQUIM, G. V. Flutuação populacional de ácaros e caracterização de sintomas de Calacarus heveae em clones de seringueira (Hevea brasiliensis Muell. Arg.) cultivados em Jaboticabal - SP. 1994. 99f. Monografia (Graduação) - Curso de Graduação em Agronomia, Universidade do Noroeste do Estado de São Paulo, Jaboticabal, 1994.

R. Árvore, Viçosa-MG, v.32, n.2, p.365-376, 2008 
HERNANDES, F. A.; FERES, R. J. F. Diversidade e sazonalidade de ácaros (Acari) em seringal (Hevea brasiliensis, Muell. Arg.) no Noroeste do Estado de São Paulo. Neotropical Entomology, v.35, n.4, p.523-535, 2006.

JEPPSON, L. R. et al. Mites injurious to economic plants. Berkeley: University of California Press, 1975. 614p.

LIMA, D. U. et al. Avaliação sazonal da produção de borracha e da dinâmica de carboidratos solúveis em plantas de seringueira (Hevea brasiliensis Müll. Arg.) cultivadas em Lavras, Minas Gerais. Revista Árvore, v.26, n.3, p.377-383, 2002.

McMURTRY, J. A.; CROFT, B.A. Life-styles of phytoseiid mites and their roles in biological control. Annual Review of Entomology, v.42, p.291-321, 1997.

R. Árvore, Viçosa-MG, v.32, n.2, p.365-376, 2008
PEREIRA, F. F. et al. Primeiro registro de Oligonychus yothersi (McGregor) Acari: Tetranychidae) em Eucalyptus grandis Hill. Ex Maiden no Brasil. Revista Árvore, v.29, n.4, p.657-659, 2005.

TANZINI, M. R. et al. An epizootic of Calacarus heveae (Acari: Eriophyidae) caused by Hirsutella thompsoni on rubber trees.

Experimental and Applied Acarology, v.24, p.141-144, 2000.

VIEIRA, M. R.; GOMES, E. C. Sintomas, desfolhamento e controle de Calacarus heveae Feres. 1992 (Acari: Eriophyidae) em seringueira (Hevea brasiliensis Muell. Arg.). Cultura Agronômica, v.8, n.1, p.53-71, 1999. 